\title{
Expansão Rápida da Maxila: análise da densidade radiográfica da sutura palatina mediana e sua cor- relação nos estágios de neoformação óssea, por meio de imagem digitalizada
}

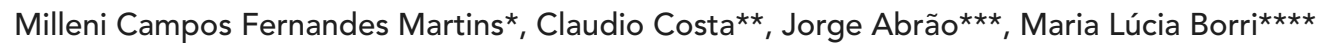

\section{Resumo}

Objetivo: avaliar a neoformação óssea da sutura palatina mediana em diferentes fases do procedimento de expansão rápida da maxila por meio de imagem digitalizada e comparar a densidade radiográfica das diferentes áreas selecionadas ao longo dessa sutura nos períodos estabelecidos. Métodos: a amostra foi constituída por 23 indivíduos, na faixa etária de 9 a 12 anos, que foram submetidos à expansão rápida da maxila de acordo com um protocolo que consistiu, inicialmente, em uma volta completa no parafuso, seguida por $1 / 4$ de volta pela manhã e $1 / 4$ de volta à noite, durante duas semanas. Foram obtidas 69 radiografias oclusais em diferentes momentos do procedimento, as quais passaram pelo processo de digitalização via scanner e, posteriormente, foram submetidas ao programa Digora for Windows 5.6, para a realização das leituras de densidade radiográfica. Resultados: em todas as fases do procedimento, os valores obtidos dos níveis de cinza foram, em média, diferentes entre si, comprovando uma recuperação óssea parcial da sutura palatina mediana. Conclusões: os resultados revelaram que são necessários mais de três meses para a completa recuperação sutural após a expansão rápida da maxila e que o método utilizado para a leitura das densidades mostrou-se fiel, devido à sua capacidade de exibir detalhes. Portanto, conclui-se que o expansor deve ser removido somente após a total recuperação óssea sutural, a fim de evitar as recidivas.

Palavras-chave: Ortodontia. Sutura. Técnica de expansão palatina. Diagnóstico por imagem.

\section{INTRODUÇÃO}

A expansão rápida da maxila já é um procedimento consagrado na prática ortodôntica. Segundo Haas $^{5}$, sua maior indicação é na correção das mordidas cruzadas posteriores causadas por deficiência transversal da maxila. Essa deficiência, somada à ausência de discrepância sagital entre as bases apicais, ocorre devido a uma alteração na conformação do arco dentário superior, que assume um aspecto triangular, caracterizando a atresia maxilar ${ }^{1,2,18,19}$.

\footnotetext{
* Especialista em Ortodontia pela Universidade Camilo Castelo Branco - Unicastelo. Mestre em Ciências Radiológicas pelo Departamento de Diagnóstico por Imagem da Universidade Federal de São Paulo - Unifesp.

** Livre-docente em Diagnóstico Bucal pela FOUSP. Professor associado livre-docente da disciplina de Radiologia da FOUSP.

*** Livre-docente em Ortodontia pela FOUSP. Professor associado livre-docente da disciplina de Ortodontia da FOUSP.

**** Doutora em Radiologia pelo Departamento de Diagnóstico por Imagem da Unifesp. Professora responsável do setor de Tomografia Computadorizada da Unifesp.
} 
$\mathrm{O}$ arco superior atrésico sempre foi de grande interesse para os pesquisadores, que têm se preocupado com a relação dentomaxilomandibular.

A intervenção precoce é considerada a melhor opção para tratamento, permitindo resultados excelentes. Nessa fase, o efeito ortopédico é bastante presente, pois, com o avanço da maturidade óssea, esse efeito diminui, devido à consequente compensação dentária. Sendo assim, para que a correção das mordidas cruzadas posteriores seja realizada com sucesso, o procedimento ortodôntico eleito é a expansão rápida da maxila. Para que esse procedimento ocorra, é fundamental que a força aplicada aos dentes e ao processo alveolar exceda o limite necessário para a movimentação ortodôntica, promovendo, assim, a separação dos ossos maxilares. $\mathrm{Mew}^{9}$ comentou que o grande inconveniente do efeito ortodôntico é o excesso de vestibularização dentoalveolar, que promove pouca estabilidade pós-contenção. Por esse motivo, deve-se considerar a necessidade de sobrecorreção do arco atrésico para compensar a subsequente e inevitável verticalização dos segmentos dentoalveolares posteriores ${ }^{17}$.

Para corrigir as atresias maxilares, a expansão rápida da maxila é, hoje, o recurso clínico mais utilizado, independentemente da técnica empregada. A literatura é unânime em reconhecer a efetividade dos expansores como instrumento para romper as suturas maxilares.

Ainda na metade do século passado, $\mathrm{Haas}^{5}$ notou que o diastema entre os incisivos centrais superiores ocorria mediante a abertura da sutura palatina mediana. Essa abertura foi comprovada por meio de um exame radiográfico oclusal, onde se visualizou uma imagem radiolúcida ampla com formato triangular ou em forma de "V", tendo sua base voltada para a região anterior. Sendo assim, o autor descreveu um aparelho dentomucossuportado capaz de conferir maior rigidez, favorecendo maior transferência das forças de ativação às bases ósseas para promover a estabilidade ortopédica pós-expansão. Mais tarde, sugerindo modificações nos expansores convencionais, Biederman ${ }^{4}$ relatou o aparelho expansor sem a presença do acrílico, sendo denominado de aparelho dentossuportado. $\mathrm{O}$ autor identificou a indesejável inflamação inerente aos aparelhos confeccionados com acrílico, por dificultarem a higienização devido à impacção alimentar. Sua ativação era feita de maneira semelhante à de outros aparelhos e os resultados relataram uma vestibularização do segmento posterior.

Com a finalidade de diagnóstico, Santos-Pinto e Henriques ${ }^{13}$ afirmaram que a radiografia oclusal assume um papel muito importante após o procedimento de expansão rápida da maxila, pois é por meio dessa que se obtém a imagem da sutura palatina mediana.

$\mathrm{Mol}^{10}$ acredita que a radiografia possibilita a orientação da conduta terapêutica, permitindo seu acompanhamento, desde que ocorra uma padronização na obtenção das imagens. Considerando que mudanças nos ângulos vertical e horizontal podem produzir erros de projeção irreversíveis na imagem, a padronização da distância foco-filme se torna fundamental.

Portanto, diante da possibilidade de recidivas que podem ocorrer com o aumento transversal do arco superior, e sendo a recuperação óssea da sutura palatina mediana um dos fatores que podem contribuir para a estabilidade do tratamento, as leituras de densidade atuam como um recurso, oferecido pela digitalização das imagens, utilizado para fornecer medidas mais precisas dos índices de escala dos tons de cinza. Essas leituras possibilitam o acompanhamento da evolução das neoformações ósseas, de forma padronizada, em diferentes períodos de tempo. Uma das suas vantagens é a possibilidade de se medir a densidade de uma área selecionada ${ }^{11,13}$. Outra vantagem bastante significativa é a inclusão de tarefas matemáticas que esses programas permitem, complementando o diagnóstico e favorecendo ainda mais sua interpretação ${ }^{14}$. Todas essas vantagens permitem melhorar a precisão do diagnóstico, embora esses sistemas digitais apresentem um custo alto, que 
restringe sua utilização a centros de pesquisas.

Dessa maneira, estudos utilizando imagens digitais e programas específicos de análise da densidade para a avaliação da neoformação óssea da sutura palatina mediana após a expansão rápida da maxila vêm sendo publicados, com finalidade de evitar os efeitos indesejáveis que podem ocorrer quando se faz a remoção do aparelho disjuntor antes do término do processo de ossificação e reorganização sutural. Nesse sistema, o diagnóstico fundamenta-se na detecção das diferentes densidades entre imagens sequenciadas que representam as alterações da neoformação óssea.

$\mathrm{Melo}^{7}$ mensurou as densidades ópticas da sutura palatina mediana, por meio de um sistema de radiografia digital por placa fotoativada, em pacientes submetidos à expansão rápida da maxila, comparando-as e correlacionando-as com a radiografia oclusal convencional. Concluiu que os resultados encontrados nas imagens digitais foram compatíveis com os obtidos nas radiografias oclusais convencionais.

Outro estudo com a finalidade de avaliar a maturação óssea na região da sutura palatina mediana após a expansão rápida da maxila, por meio da variação de densidade óptica utilizando o programa DentScan Dentview (Apica Eng. Ltda - Dental Technologies, Nesher, Israel), foi realizado por Simões, Araújo e Bittencourt ${ }^{20}$. Os autores compararam os valores pré-expansão com os encontrados na fase de contenção e obtiveram como resultado que a maturação óssea ocorreu precocemente na região anterior em relação à posterior.

Verificando-se a possibilidade de recidiva destes tratamentos, devemos considerar o tempo de contenção. Esse período é bastante controverso, embora seja unânime a sua necessidade, pois o tecido ósseo apresentado na sutura palatina mediana imediatamente após a expansão mostra-se como tecido conjuntivo desorganizado altamente vascular, o qual posteriormente será substituído por um tecido ósseo imaturo. Portanto, abordando o tempo de contenção após a expansão rápida da maxila, vários autores sugerem um período de três a seis meses para se garantir a estabilidade, a reorganização da sutura e a dissipação das forças residuais acumuladas. Além disso, após a retirada do aparelho expansor, seria importante a instalação de uma placa removível por mais seis meses para a estabilização da oclusão $0^{3,19}$.

Um dos fatores mais importantes para a estabilidade dos resultados da expansão rápida é, certamente, a completa formação óssea da sutura palatina mediana. Por isso, o acompanhamento da recuperação óssea sutural por meio do exame radiográfico oclusal tem sido bastante estudado ${ }^{6}$. Segundo Melsen ${ }^{8}$, no primeiro mês de controle após a expansão, a imagem radiográfica mostrou que já havia se iniciado o processo de neoformação óssea, entretanto ainda de forma difusa. Ao longo do tempo, essa neoformação continuou progredindo até a sua recuperação completa. Portanto, como forma de se evitar as recidivas, seria imprescindível a neoformação óssea completa da sutura palatina mediana ${ }^{15,23}$. Outro fator relacionado com a estabilidade é a sobre-expansão. De acordo com Timms ${ }^{22}$, uma vez que essa é influenciada pela inclinação dos processos alveolares, seria indicada uma sobrecorreção de $1 / 3$ a 1/2 a mais de expansão e a permanência de contenção por um período mais prolongado.

Preocupados também com a estabilidade pós-expansão, em um estudo radiográfico recente, Silva Filho et al. ${ }^{16}$ analisaram a ossificação da sutura palatina mediana de pacientes submetidos à expansão rápida da maxila, com o objetivo de comprovar a validade de se avaliar a neoformacão óssea sutural por meio do exame radiográfico oclusal. Como resultados, obtiveram que são necessários mais de três meses após o procedimento para que o processo de ossificação sutural se processe. Dessa forma, sugerem um diagnóstico detalhado, sobrecorreção imediata e prolongada contenção pós-expansão, com uma mecânica subsequente que respeite as dimensões transversais conseguidas com a expansão rápida da maxila. 


\section{PROPOSIÇÃO}

Diante da possibilidade de recidivas que podem ocorrer com o aumento transversal do arco superior, e sendo a neoformação óssea na região da sutura palatina mediana um dos fatores que podem contribuir para a estabilidade do tratamento, a proposta deste estudo foi acompanhar a evolução da neoformacão óssea da sutura palatina mediana de pacientes submetidos à expansão rápida da maxila, por meio de imagem digitalizada, e comparar as densidades radiográficas obtidas nos períodos estabelecidos.

\section{MATERIAL E MÉTODOS}

A amostra estudada neste trabalho constituiuse de 23 indivíduos com idades entre 9 e 12 anos, sendo o estudo realizado parte no Departamento de Diagnóstico por Imagem da Unifesp e parte na CETAO (Instituição de Ensino Superior - Extensão e Pós-Graduação em Odontologia). Todos foram tratados com o procedimento de expansão rápida da maxila, sendo submetidos ao mesmo protocolo de ativação, por meio dos aparelhos expansores dentossuportados Hyrax (Fig. 1A) e McNamara (Fig. 1B).

O procedimento da expansão teve duração de duas semanas, sendo que as ativações iniciaram com uma volta completa no parafuso uma semana após a instalação do disjuntor, continuando com duas ativações diárias de 1/4 de volta pela manhã e $1 / 4$ de volta à noite. Uma vez concluída a disjunção, o parafuso expansor foi fixado, para que não ocorresse nenhuma movimentação. Com a finalização dessa fase, iniciou-se a fase de contenção pós-expansão, a qual teve duração de três meses.

Os diferentes momentos analisados durante o procedimento foram divididos em: Fase I - antes da expansão (Fig. 2A); Fase II - término da expansão e fixação do parafuso (Fig. 2B); e Fase III - três meses após o término da expansão (Fig. 2C).

Durante essas fases, as radiografias oclusais foram obtidas e posteriormente digitalizadas. Para a
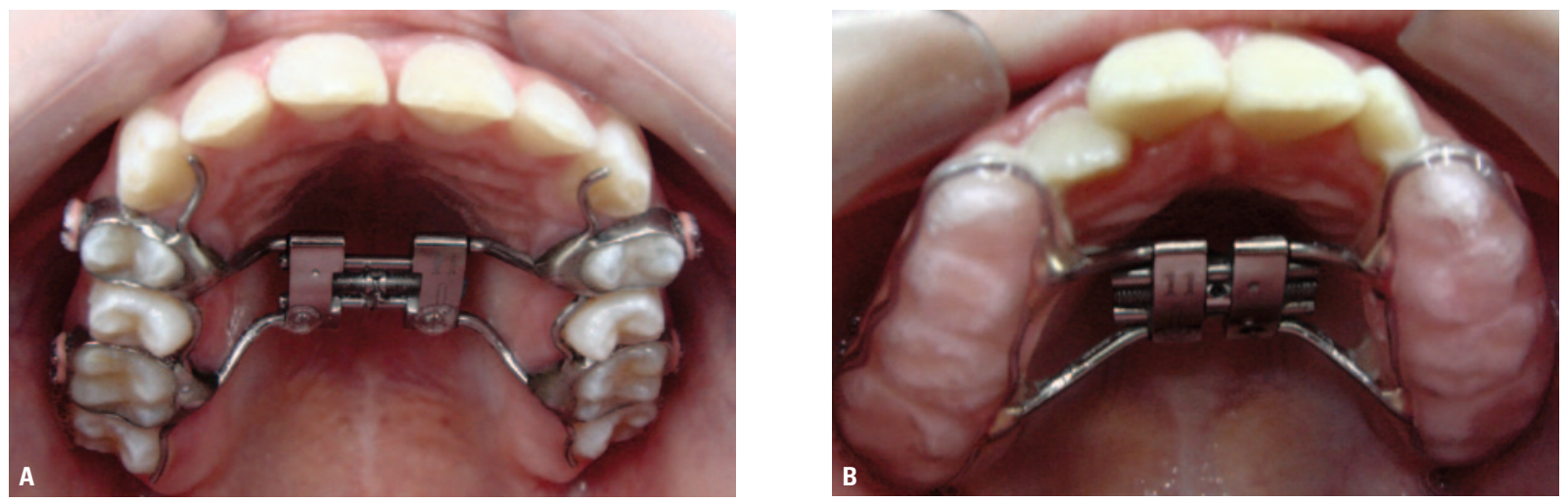

FIGURA 1 - A) Disjuntor dentossuportado Hyrax e B) disjuntor dentossuportado McNamara.
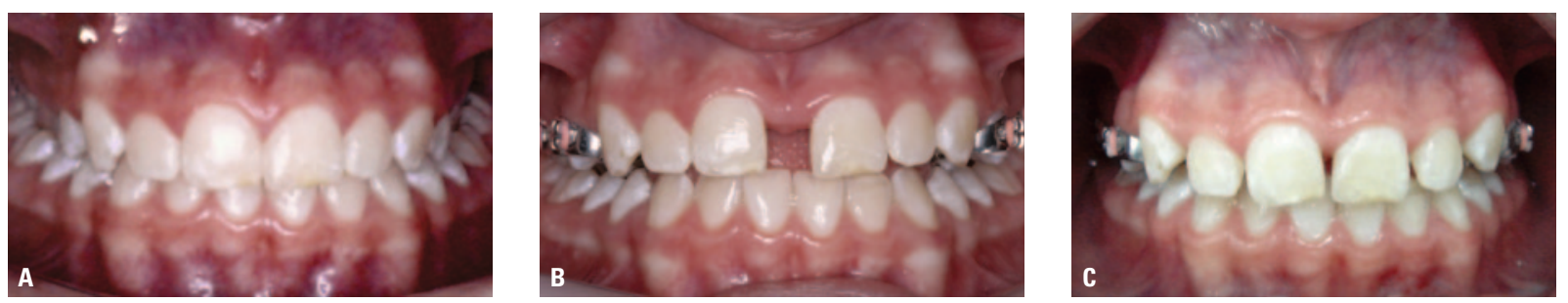

FIGURA 2 - A) Fase I - antes da expansão rápida da maxila. B) Fase II - após a expansão rápida da maxila. C) Fase III - três meses após a expansão rápida da maxila. 
obtenção dessas imagens, utilizou-se um aparelho de raios X Spectro II $^{\circledR}$ Dabi-Atlante, com tempo de exposição de 1s, filmes oclusais Kodak Insight e um posicionador para técnica oclusal com inclinação de $65^{\circ}$ a fim de determinar a distância foco-filme. Com a finalidade de padronização, um corpo-de-prova radiopaco, representado pelo número 1 , foi instalado na radiografia antes da incidência radiográfica, visando a equalização das leituras de densidade e a eliminação das variações relacionadas com a temperatura e a concentração das soluções químicas durante seu processamento, bem como variáveis referentes ao tipo de scanner utilizado. Todas as radiografias foram reveladas por processamento automático, em uma processadora AT 2000 PLUS (Air Techniques, EUA), com

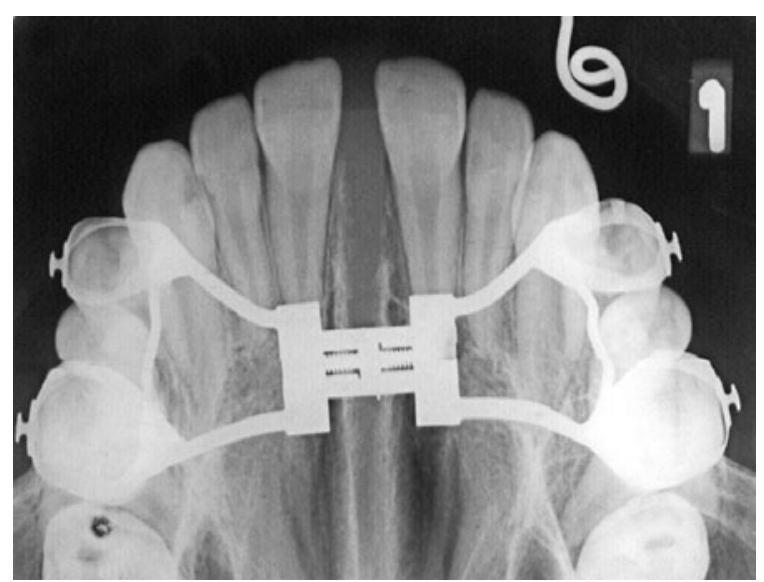

FIGURA 3 - Imagem digitalizada da sutura palatina mediana após seu rompimento (Fase II). solução química Kodak a $30^{\circ} \mathrm{C}$. Em seguida, essas imagens foram digitalizadas (Fig. 3) em um scanner SCANION A3 (ÍON, Belo Horizonte/MG) com resolução de 300dpi e submetidas a um programa específico para leituras de imagens digitais (Digora for Windows 5.6, Digital Dental, Reino Unido).

Essas imagens foram realçadas em suas características de brilho, contraste e densidade (recursos oferecidos pelo programa utilizado). Após esse procedimento, duas áreas de interesse foram demarcadas na região da sutura palatina mediana e uma terceira área no corpo-de-prova que foi instalado nas radiografias conforme descrito anteriormente. A primeira área, denominada "A", situou-se na região anterior da sutura palatina mediana (Fig. 4A). A segunda área, denominada "B", localizouse na região posterior da sutura palatina mediana (Fig. 4B); e a terceira área, denominada "C", foi localizada dentro do corpo-de-prova (Fig. 4C).

Após a obtenção dos valores médios de densidade nos três momentos estabelecidos, os mesmos foram submetidos a tratamento estatístico.

\section{RESULTADOS}

Para a análise estatística de todas as informações coletadas nesta pesquisa foi utilizada a Análise de Variância (ANOVA), juntamente com as comparações múltiplas pelo método de Bonferroni. Em todas as conclusões obtidas por meio das análises inferenciais foi utilizado o nível de
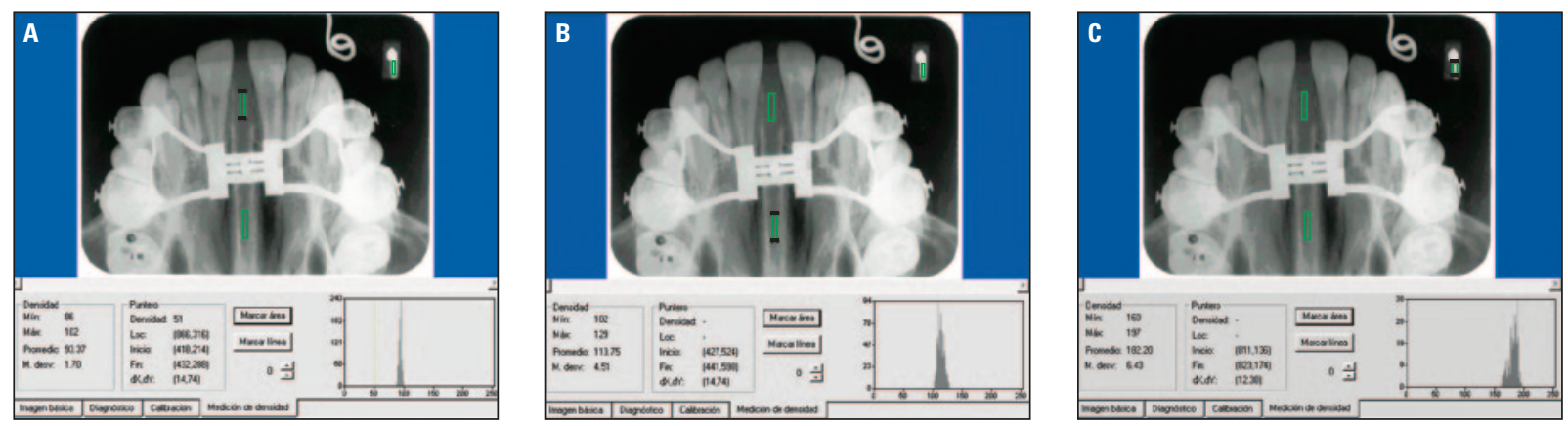

FIGURA 4 - A) Localização da área "A", situada na região anterior ao longo da sutura palatina mediana. B) Localização da área "B", situada na região posterior ao longo da sutura palatina mediana. C) Localização da área "C", situada dentro do corpo-de-prova instalado no filme antes da incidência radiográfica. 
significância igual a 5\%. Assim, os resultados obtidos foram expressos na forma de tabelas e gráficos.

De acordo com a tabela 1, os resultados mostraram que, em todas as fases do procedimento, os valores obtidos dos níveis de cinza, ou seja, das densidades, são, em média, diferentes entre si, sendo que, na fase inicial, a densidade mostra-se com valor maior, diminuindo imediatamente na fase intermediária e aumentando na fase final, com tendência a atingir o valor médio inicial.

Sendo assim, analisando-se a tabela 1 e o gráfico 1, as medidas da área "A" corresponderam, em média, a quase $80 \%$ do valor inicial, enquanto a área "B" correspondeu, em média, a 75\% do valor inicial. Portanto, analisando-se as áreas "A" e "B" nos diferentes momentos da pesquisa, verificouse que, após os três meses de contenção, o valor médio obtido de densidade não foi equivalente ao observado no período pré-expansão.
Os resultados inferenciais revelaram que os níveis médios de cinza das áreas " $\mathrm{A}$ " $(\mathrm{p}<0,001)$ e " $\mathrm{B}$ " $(\mathrm{p}<0,001)$ não são estatisticamente os mesmos ao longo dos três momentos da coleta. Conforme mostram as tabelas 2 e 3 , as comparações entre os momentos inicial e intermediário para as áreas "A" e "B" revelaram os mesmos resultados. A densidade na fase inicial, quando comparada com a fase

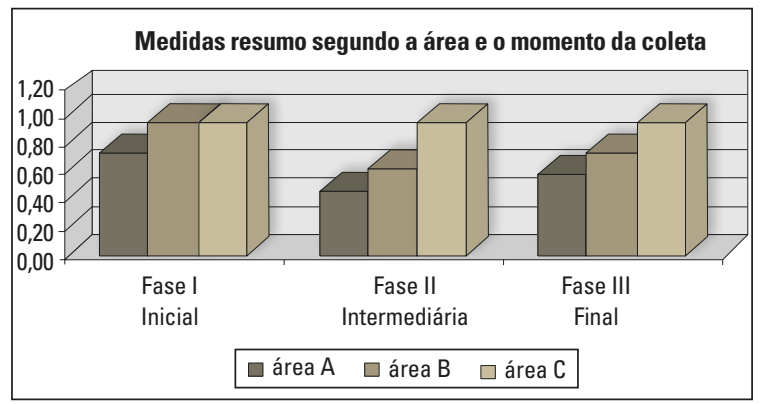

GRÁFICO 1 - Distribuição dos níveis de cinza das áreas "A", "B" e "C", nas fases I, II e III.

TABELA 1 - Medidas-resumo dos níveis de cinza, segundo a área e o momento da coleta.

\begin{tabular}{|c|c|c|c|c|c|c|}
\hline \multirow[b]{2}{*}{ Área } & \multicolumn{6}{|c|}{ Medidas-resumo } \\
\hline & Momento & Média & Mediana & Mínimo & Máximo & d.p. \\
\hline \multirow{3}{*}{ Área A } & inicial & 0,75 & 0,75 & 0,52 & 1,04 & 0,14 \\
\hline & intermediária & 0,48 & 0,48 & 0,34 & 0,68 & 0,08 \\
\hline & final & 0,59 & 0,56 & 0,47 & 0,83 & 0,09 \\
\hline \multirow{3}{*}{ Área B } & inicial & 1,00 & 0,95 & 0,48 & 1,59 & 0,27 \\
\hline & intermediária & 0,65 & 0,63 & 0,40 & 0,95 & 0,13 \\
\hline & final & 0,75 & 0,75 & 0,54 & 0,98 & 0,11 \\
\hline
\end{tabular}

TABELA 2 - Resultados das comparações entre os momentos para a área "A" pelo método de Bonferroni.

\begin{tabular}{|c|c|c|}
\hline \multicolumn{2}{|c|}{ Momentos comparados } & Conclusão \\
\hline inicial & intermediário & inicial $>$ intermediário $(p<0,001)$ \\
\hline inicial & final & inicial $>$ final $(p<0,001)$ \\
\hline intermediário & final & intermediário $<$ final $(p=0,001)$ \\
\hline
\end{tabular}

TABELA 3 - Resultados das comparações entre os momentos para a área "B" pelo método de Bonferroni.

\begin{tabular}{|c|c|c|}
\hline \multicolumn{2}{|c|}{ Momentos comparados } & Conclusão \\
\hline inicial & intermediário & inicial > intermediário $(p<0,001)$ \\
\hline inicial & final & inicial > final $(p=0,003)$ \\
\hline intermediário & final & intermediário < final $(p=0,010)$ \\
\hline
\end{tabular}


intermediária, é maior. Os resultados revelaram que a densidade na fase inicial é maior que a densidade na fase final, e que a densidade na fase intermediária é menor que a densidade na fase final.

\section{DISCUSSÃO}

A partir da introdução da expansão rápida da maxila ${ }^{1}$, esse método de tratamento passou a ser testado, sendo eleito para a correção nos casos de deficiência transversal da maxila. Essa deficiência do arco dentário superior assume um aspecto triangular, caracterizando a mordida cruzada posterior. Com a finalidade de corrigir as atresias desse arco, a expansão rápida da maxila foi incorporada integralmente na mecanoterapia moderna, independentemente da técnica empregada. Autores como Angell ${ }^{1}$; Timms ${ }^{22}$; Silva Filho et al. ${ }^{17}$; Silva Filho, Capelozza Filho ${ }^{18}$; Reed, Gosh e Nan$\mathrm{da}^{12}$ são unânimes em reconhecer a efetividade dos expansores como instrumento para romper as suturas maxilares - por meio de forças pesadas que ocorrem com a ativação rápida do parafuso -, permitindo, assim, que o aparelho disjuntor faça parte do rol dos aparelhos ortopédicos.

É comprovado na literatura que os aparelhos expansores são eficazes e, segundo Reed, Gosh, Nanda $^{12}$; Siqueira, Almeida e Henriques ${ }^{21}$, os efeitos dentoesqueléticos produzidos, embora apresentem diferenças, são estatisticamente insignificantes, não havendo qualquer superioridade do aparelho colado com cobertura oclusal em relação ao bandado. Por esse motivo, no presente estudo utilizamos dois tipos de aparelhos expansores dentossuportados (Hyrax e McNamara).

Dentre as vantagens do sistema digital, Sarmento et al. ${ }^{14}$ salientam que a medida da densidade de uma área selecionada é bastante importante, sendo representada por valores numéricos que correspondem ao mínimo, ao máximo e à média dos tons de cinza. Já a ampliação da imagem digitalizada ocorre quando se deseja mensurar a densidade em determinadas áreas, e o seu valor numérico corresponde à média dos tons de cinza.
Sendo assim, a quantidade de nuances de cinza no momento em que se determina a correspondência numérica irá determinar uma capacidade maior de mostrar os detalhes.

Dessa maneira, autores como Mello ${ }^{7}$; Simões, Araújo e Bittencourt ${ }^{20}$ realizaram seus estudos utilizando imagens digitais e programas específicos de análise da densidade (para a avaliação da neoformação óssea da sutura palatina mediana após a expansão rápida da maxila), com finalidade de evitar os efeitos indesejáveis que podem ocorrer quando se faz a remoção do aparelho disjuntor antes do término do processo de ossificação e reorganização sutural.

Para a realização da análise descritiva de cada área, as medidas de densidade na região da sutura palatina mediana foram avaliadas em três fases da terapia expancionista, sendo aferidas na fase I (que corresponde ao início do tratamento e antes da ativação), na fase II (que representa o período imediatamente após a expansão rápida da maxila) e na fase III (período de contenção correspondente aos três meses subsequentes ao término da ativação). Conforme mostram a tabela 1 e o gráfico 1 , as medidas da área "A" na fase inicial possuem média total equivalente a 0,75 , diminuindo para 0,48 logo após a abertura da sutura e aumentando para 0,59 após o terceiro mês de contenção correspondendo, em média, a quase $80 \%$ do valor inicial. Já a área "B", na fase inicial, possui média total equivalente a 1,0 , diminuindo para 0,65 logo após a abertura da sutura e aumentando para 0,75 após o terceiro mês de contenção - correspondendo, em média, a $75 \%$ do valor inicial. A análise estatística, feita de forma descritiva, verificou que as médias inicial e final são diferentes, com significância de 5\%.

Dessa forma, foi observado que o processo de neoformação óssea não se completou até o terceiro mês de acompanhamento pós-contenção. Portanto, tal constatação confirma a necessidade da obtenção de uma radiografia oclusal da maxila por volta do terceiro mês de contenção, pois essa 
imagem determinará a condição da sutura e, por consequência, a época de finalização do período de contenção e remoção do aparelho disjuntor.

Analisando as áreas "A" e "B" (Tab. 1) nos diferentes momentos da pesquisa, verificou-se uma queda logo após a abertura da sutura palatina mediana e um aumento da densidade após os três meses de contenção, porém o valor alcançado não foi equivalente ao observado no período pré-expansão. Além disso, nesse estudo, observouse que a maturação óssea ocorreu precocemente na região anterior em relação à posterior, estando de acordo com os resultados de Simões, Araújo e Bittencourt ${ }^{20}$.

Embora a literatura seja unânime em afirmar que um período mínimo de três meses é necessário para promover o restabelecimento ósseo da sutura palatina mediana ${ }^{2,5,15,18,19}$, a análise dos dados obtidos mostra que a densidade ao final do terceiro mês de contenção não correspondeu à inicial, tornando-se evidente que a formação óssea não se completou. Portanto, um tempo maior de contenção é sugerido para recuperação óssea nessa área. Os resultados obtidos nesse estudo são semelhantes aos de Simões, Araújo e Bittencourt ${ }^{20}$ e estão de acordo com os de Mello ${ }^{7}$ e Silva Filho et al. ${ }^{16}$, que afirmam ser necessário um período de contenção maior do que três meses para o completo restabelecimento dessa sutura.

\section{CONCLUSÃO}

De acordo com os resultados obtidos, observou-se que ocorreram diferenças significativas nos valores médios dos níveis de cinza. Com isso, concluiu-se que a neoformação óssea da sutura palatina mediana sofreu variações em função do tempo, nas várias fases analisadas, apresentando uma ossificação incompleta no período de três meses de contenção, o qual foi insuficiente para a recuperação completa dessa sutura. Sendo assim, sugere-se a remoção do aparelho expansor somente depois que a reorganização da sutura estiver completamente concluída, e que o tempo de contenção após a expansão rápida seja maior com a finalidade de controlar as indesejáveis recidivas, beneficiando, portanto, a estabilidade do tratamento. Concluiu-se também que, por meio do estudo comparativo das densidades radiográficas, a neoformação óssea sutural ocorreu primeiramente na região anterior, em relação à região posterior, e que a leitura das densidades utilizando o software Digora for Windows 5.6 revela com clareza a definição de detalhes. $\mathrm{O}$ uso das imagens digitalizadas na análise da densidade radiográfica é uma ferramenta que permite uma interpretação minuciosa da neoformação óssea da sutura palatina mediana, mostrando detalhes muitas vezes difíceis de serem observados a olho nu. Dessa forma, com o avanço tecnológico, espera-se que o problema do alto custo seja solucionado e que esses equipamentos se tornem cada vez mais acessíveis aos profissionais da área, a fim de se obter uma melhor estimativa quanto ao tempo de contenção pós-tratamento, promovendo uma estabilidade mais efetiva após o procedimento de expansão rápida da maxila.

Enviado em: fevereiro de 2009 Revisado e aceito: agosto de 2009

Milleni Campos Fernandes Martins Av. Rouxinol, 1041, cj. 1610 Moema CEP: 04.516-001 - São Paulo / SP

E-mail: milleni.martins@terra.com.br 


\title{
Rapid Maxillary Expansion: Radiographic analysis of the midpalatal suture density and its correlation in the osteogenesis stages by digital image
}

\begin{abstract}
Aim: To evaluate the new bone formation of the midpalatal suture in different phases of the rapid maxillary expansion procedure by means of the scanned image and to compare the radiographic density of the different selected areas along this suture in predetermined periods. Methods: The sample consisted of 23 individuals, aged from 9 to 12 years who underwent rapid maxillary expansion in accordance with a protocol which consisted initially of a full turn on the screw, followed by $1 / 4$ turn by morning and $1 / 4$ turn at night for two weeks. Were obtained 69 occlusal radiographs at different phases of the procedure, which passed through the scanning process via scanner and then were subjected to the software Digora for Windows 5.6, to perform the readings of radiographic density. Results: In all phases of the procedure, the values of gray levels were, on average, different from each other, showing partial bone healing of the midpalatal suture. Conclusions: The results revealed that are necessary more than three months for complete sutural recovery after the rapid maxillary expansion and that the method used to read the densities proved to be trustworthy, because of its ability to display details. Therefore, it can be concluded that the expander should be removed only after full sutural bone recovery in order to avoid relapses.
\end{abstract}

Keywords: Orthodontics. Sutures. Palatal expansion technique. Orthodontics. Imaging diagnostic.

\section{REFERÊNCIAS}

1. ANGELL, E. H. Treatment of irregularity of the permanent or adult teeth. Part. 1. Dental Cosmos, Philadelphia, v. 1, no. 10, p. 540-544, May 1860.

2. ANGELL, E. H. Treatment of irregularity of the permanent or adult teeth. Part. 2. Dental Cosmos, Philadelphia, v. 1, no. 10, p. 599-600, June 1860.

3. BELL, R. A. A review of maxillary expansion in relation to rate of expansion and patient's age. Am. J. Orthod., St. Louis, v. 81 , p. $132-137,1982$.

4. BIEDERMAN, W. A hygienic appliance for rapid expansion. J. Pract. Orthod., Hempstead, v. 2, no. 2, p. 67-70, Feb. 1968.

5. HAAS, A. J. Rapid expansion on the maxillary dental arch and nasal cavity by opening the midpalatal suture. Angle Orthod. Appleton, v. 31, no. 2, p. 73-90, Apr. 1961.

6. INOUE, N.; OHYAMA, K.; ISHIGURO, K.; AZUMA, M.; OZAKI, T.; KOSUGI, R. Radiographic observation of rapid expansion of human maxilla. Bull. Tokyo Med. Dent. Univ., Tokyo, v. 17, no. 3, p. 149-161, Sept. 1970.

7. MELO, M. F. B. Avaliação da densidade óptica da sutura palatina mediana por meio do sistema de radiografia digital por placa fotoativada em pacientes submetidos a disjunção palatina. 2003. Dissertação (Mestrado)-Faculdade de Odontologia, Universidade de São Paulo, São Paulo, 2003.

8. MELSEN, B. A histological study of the influence of sutural morphology and skeletal maturation on rapid palatal expansion in children. Trans. Eur. Orthod. Soc., London, p. 499-507, 1972.

9. MEW, J. Relapse following maxillary expansion: A study of twenty-five consecutive cases. Am. J. Orthod., St. Louis, v. 83, no. 1, p. 56-61, Jan. 1983

10. MOL, A. Image processing tools for dental applications. Dent. Clin. North Am., Philadelphia, v. 44, no. 2, p. 299-318, 2000.

11. OlIVEIRA, A. E.; ALMEIDA, S. M.; PAGANINI, G. A.; HAITER NETO, F.; BÓSCOLO, F. N. Comparative study of two digital radiographic storage phosphor system. Braz. Dent. J., Ribeirão Preto, v. 11, n. 2, p. 11-116, out. 2000.

12. REED, N.; GHOSH, J.; NANDA, R. S. Comparison of treatment outcomes with banded and bonded RPE appliances. Am. J. Orthod., St. Louis, v. 116, no. 1, p. 31-40, July 1999.

13. SANTOS-PINTO, C. C. M.; HENRIQUES, J. F. C. Expansão rápida da maxila: preceitos clínicos e radiográficos. Rev. Odontol. USP., São Paulo, v. 4, n. 2, p. 164-166, 1990.
14. SARMENTO, V. A. et. al. Entendendo a imagem digitalizada. Rev. Odonto Ciência, Porto Alegre, v. 14, n. 27, p. 171-178, 1999.

15. SILVA FILHO, O. G. Expansão Rápida da Maxila: um ensaio sobre a sua instabilidade. R. Dental Press Ortodon. Ortop. Facial, Maringá, v. 8, n. 1, p. 17-36, jan./fev. 2003.

16. SILVA FILHO, O. G. et al. Ossificação da sutura palatina mediana após o procedimento de expansão rápida da maxila: estudo radiográfico. R. Dental Press Ortodon. Ortop. Facial, Maringá, v. 13, n. 2, p. 124-131, mar./abr. 2008.

17. SILVA FILHO, O. G. et al. Rapid maxillary expansion in the deciduous and mixed dentition evaluated through posteroanterior cephalometric analysis. Am. J. Orthod. St. Louis, v. 107, no. 3, p. 268-275, Mar. 1995.

18. SILVA FILHO, O. G.; CAPELOZZA FILHO, L. Expansão rápida da maxila: considerações gerais e aplicação clínica. Parte I. R. Dental Press Ortodon. Ortop. Facial, Maringá, v. 2, no. 3, p. 88-102, maio/jun. 1997.

19. SILVA FILHO, O. G.; CAPELOZZA FILHO, L. Expansão rápida da maxila. Parte II. R. Dental Press Ortodon. Ortop. Facial Maringá, v. 2, no. 4, p. 86-108, jul./ago. 1997.

20. SIMÕES, F. X. P. C.; ARAÚJO, T. M.; BITTENCOURT, M. A. V. Avaliação da maturação óssea na sutura palatina mediana, após expansão rápida da maxila, por meio de imagem digitalizada. R. Dental Press Ortodon. Ortop. Facial Maringá, v. 8, n. 1, p. 59-67, jan./fev. 2003.

21. SIQUEIRA, D. F.; ALMEIDA, R. R.; HENRIQUES, J. F. C. Estudo comparativo por meio de análise cefalométrica em norma frontal dos efeitos dentoesqueléticos produzidos por três tipos de expansores palatinos. R. Dental Press Ortodon. Ortop. Facial, Maringá, v. 7, n. 6, p. 27-47, nov./dez. 2002.

22. TIMMS, D. J. An occlusal analysis of lateral maxillary expansion with midpalatal suture opening. Dent. Pract. Dent. Rec., Bristol, v. 18, no. 12, p. 435-440, 1978.

23. VASCONCELOS, M. H. F. et al. Avaliação da remodelação óssea da sutura palatina mediana com radiografia digitalizada após expansão rápida da maxila assistida cirurgicamente. In: CONGRESSO DA ASSOCIAÇÃO BRASILEIRA DE ORTODONTIA E ORTOPEDIA FACIAL, 5., 2005, Salvador. Anais... Salvador: Associação Brasileira de Ortodontia e Ortopedia Facial, 2005. p. 1-14. 